\title{
The effects of particle drift on the modulation of galactic electrons in the global heliosphere
}

\author{
R.R. Nndanganeni ${ }^{1}$ \\ Centre for Space Research, North-West University, Potchefstroom, South Africa \\ E-mail:20884648@nwu.ac.za
}

\section{M.S. Potgieter}

Centre for Space Research, North-West University, Potchefstroom, South Africa

E-mail: Marius.Potgieter@nwu.ac.za

\begin{abstract}
The fundamental process of curvature, gradient and current sheet drifts in the heliosphere is still not fully understood, especially how solar wind and magnetic field turbulence could affect the magnitude of how cosmic ray particles drift on a global scale. General consensus is that the socalled weak scattering drift is giving too large modulation effects as follows from the application of numerical drift models to observations from the Earth to the outer heliosphere. The effects of drift on the global modulation of electrons are revisited by studying different modulation scenarios using a three-dimensional steady-state numerical model for solar minimum conditions, and for both magnetic polarity epochs. A straight forward approach is followed to illustrate how reducing drift can affect the modulation of galactic electrons on a global scale. It is found that reducing drift implicitly through changing the diffusion coefficients is far more subtle a process than the strong handed approach of decreasing the drift coefficient directly. This is evident from our results that increasing perpendicular diffusion in the radial direction reduces drift effects most effectively. Enhancing perpendicular diffusion in the polar direction is also quite effective in reducing drift effects but only in the inner heliosphere, not in the middle to the outer heliosphere.
\end{abstract}

The 34th International Cosmic Ray Conference

30 July- 6 August, 2015

The Hague, The Netherland

${ }^{1}$ Speaker 


\section{Introduction}

Particle drift play an important role in the modulation of cosmic rays (CRs) in particular during solar minimum activity period. Unlike protons, where the effect of drift could extend down to very low energies, drift effects for electrons only extend to $\sim 100 \mathrm{MeV}$ because below this energy the diffusion process dominates the transport of these particles [1]. The fundamental process of curvature, gradient and current sheet drifts in the heliosphere is still not fully understood, especially how heliospheric magnetic field (HMF) turbulence could affect the magnitude of drift on a global scale; see e.g. [2,3] for recent detailed discussions on turbulence theory as applied to the modulation studies. General consensus is that weak scattering drift is giving too large effects as follows from the application of numerical drift models to solar modulation studies when compared to observations from the Earth to the outer heliosphere [4]. The effects of drift on the global modulation of electrons are revisited by studying different modulation scenarios using a three-dimensional steady-state numerical model for solar minimum conditions, and for both HMF polarity epochs. A straightforward approach is followed to illustrate how reducing drift in an implicit way can affect the modulation of galactic electrons on such a global scale.

\section{Numerical Model}

A full three-dimensional (3D) steady-state numerical model, which includes a Jovian electron source function and gradient, curvature and current sheet drifts, is used to compute galactic electron spectra at selected position in the heliosphere including the heliosheath. The modulation processes are combined in Parker's transport equation (TPE) given by:

$$
\frac{\partial f}{\partial t}=-\left(\mathbf{V}+\left\langle\mathbf{v}_{D}\right\rangle\right) \cdot \nabla f+\nabla\left(\mathbf{K}_{s} \cdot \nabla f\right)+\frac{1}{3}(\nabla \cdot \mathbf{V}) \frac{\partial f}{\partial \ln P}+Q,
$$

where $f(\mathbf{r}, P, t)$ is the omni-directional cosmic ray distribution function dependent on position $\mathbf{r}$, rigidity $P$ and time $t$. Here, $\mathbf{V}$ is the solar wind velocity, and $\mathbf{K}_{s}$ is the $3 \mathrm{D}$ diffusion tensor. It is assumed that $\partial f / \partial t=0$ which means the contribution of short-term modulation effects is neglected with the focus instead on the global spatial and spectral trends. Terms on the right hand side respectively represent convection, gradient and curvature drifts, diffusion and adiabatic energy changes with $Q$ the Jovian electron source function as given by [5]. The pitch angle averaged guiding center drift velocity for a near isotropic $\mathrm{CR}$ distribution is related to the drift coefficient $K_{D}$ as follows: $\left\langle\mathbf{v}_{D}\right\rangle=\nabla \times\left(K_{D} \mathbf{e}_{B}\right)$ with $\mathbf{e}_{B}=\mathbf{B} / B$ and where $B$ is the magnitude of the averaged background HMF, usually assumed to have a basic Parkerian geometry. The latter gives very large particle drift over the polar regions of the heliosphere so that it is a standard practice to modify it in the polar regions of the heliosphere, see e.g. $[1,3,6,7]$.

Following a simplified approach, the diffusion coefficient (DC) parallel to the averaged background HMF in terms of $P$, with $P_{0}=1 \mathrm{GV}$, and radial distance $r$, with $r_{e}=1 \mathrm{AU}$, is:

if $P<0.4 \mathrm{GV}$ :

$$
K_{\|}=K_{0} \beta\left(1+\left(r / r_{e}\right)^{c}\right) \text {, }
$$




$$
\begin{aligned}
& \text { if } P \geq 0.4 \mathrm{GV}: \\
& \mathrm{K}_{\|}=K_{0} \beta\left(P / P_{0}\right)\left(1+\left(r / r_{e}\right)^{c}\right),
\end{aligned}
$$

with $c=1.25$. Here, $\beta$ is the ratio of the particle's speed to the speed of light. The constant $K_{0}=70$ is in units of $6 \times 10^{20} \mathrm{~cm}^{2} \mathrm{~s}^{-1}$. In the outer heliosphere, with $r \geq 84 \mathrm{AU}$, the radial dependence changes, with $c=0.6$. For the diffusion perpendicular to the HMF in the radial and polar directions, it is respectively assued that

$$
K_{\perp r}=0.02 K_{\|},
$$

and

$$
K_{\perp \theta}=K_{\perp r} F(\theta)
$$

where $F(\theta)$ makes provision for $K_{\perp \theta}>K_{\perp r}$ in the off equatorial regions (see e.g. [8,9]) by using an enhancement factor $d$, the large $d$ is, the larger $K_{\perp \theta}$ becomes in the poalr regions of the heliosphere. The drift coefficient is given by

$$
\left(K_{D}\right)=\frac{\beta P}{3 B_{m}} f_{s}
$$

with $f_{s}=\frac{(\omega \tau)^{2}}{1+(\omega \tau)^{2}}$,

where $B_{m}$ is the magnitude of the HMF modified in the polar regions [7], $\omega$ is the gyrofrequency of a CR particle in the HMF and $\tau$ represents a typical time scale defined by the scattering of CRs. For the case of weak scattering, $\omega \tau \gg$ » 1.0 , so that $f_{S}=1.0$ and $K_{D}$ becomes a maximum throughout the heliosphere which has been used in numerous numerical drift models $[1,6,10,11$ and the references therein]. In most of these models, when compared to observations, it was required to make $f_{S} \approx 0.5,[12]$ and to reduce the rigidity dependence at lower energies. If the latter is applied, the drift coefficient becomes

$$
\left(K_{D}\right)=\left(K_{D}\right)_{0} \frac{\beta P}{3 B_{m}} \frac{\left(P / P_{0}^{\prime}\right)^{2}}{1+\left(P / P_{0}^{\prime}\right)^{2}},
$$

with $\left(K_{D}\right)_{0}$ a dimensionless constant ranging from 0 to 1.0 and $P_{0}^{\prime}=1 / \sqrt{10} \mathrm{GV}$. The nature of Eq. (7) is that below $\sim 1.0 \mathrm{GV}$ drift is reduced with respect to weak scattering.

An overview of the reasons why the reduction of particle drift is required in the heliosphere was given by [9]. As illustrated above, it can be done explicitly by simply assuming $f_{s}<1.0$, and/or through modifying the HMF geometry and its spatial dependence and/or in terms of its rigidity dependence. However, reducing particle drift implicitly by changing the $\mathrm{CR}$ intensity gradients in the TPE is more complicated. This can be done by simply changing any of 
the diffusion coefficients (DCs), including enhancing perpendicular diffusion in the polar direction. It was illustrated by [1] that large perpendicular diffusion in the polar regions of the heliosphere could suppress most effectively the strong latitude effects generated by strong global drifts; see also [13,14]. Perpendicular diffusion, in general, plays an important role in the heliospheric modulation of galactic CRs so that changing its value and rigidity dependence affects the effectiveness of drift. In this work, the focus is on illustrating the implicit reduction of drift through changing the diffusion coefficients. Only solar minimum conditions with the tilt angle $\alpha$ $=5^{\circ}$ is considered. Computations are done for the both magnetic polarity epochs. The termination shock (TS) is specified at 94 AU and the heliopause (HP) as heliospheric modulation boundary, is specified at $122 \mathrm{AU}$. The heliopause spectrum (HPS) for electrons [15] is specified at the heliopause position. The HPS has a power law shape at low energies with $E^{-1.55}$, where $E$ is kinetic energy.
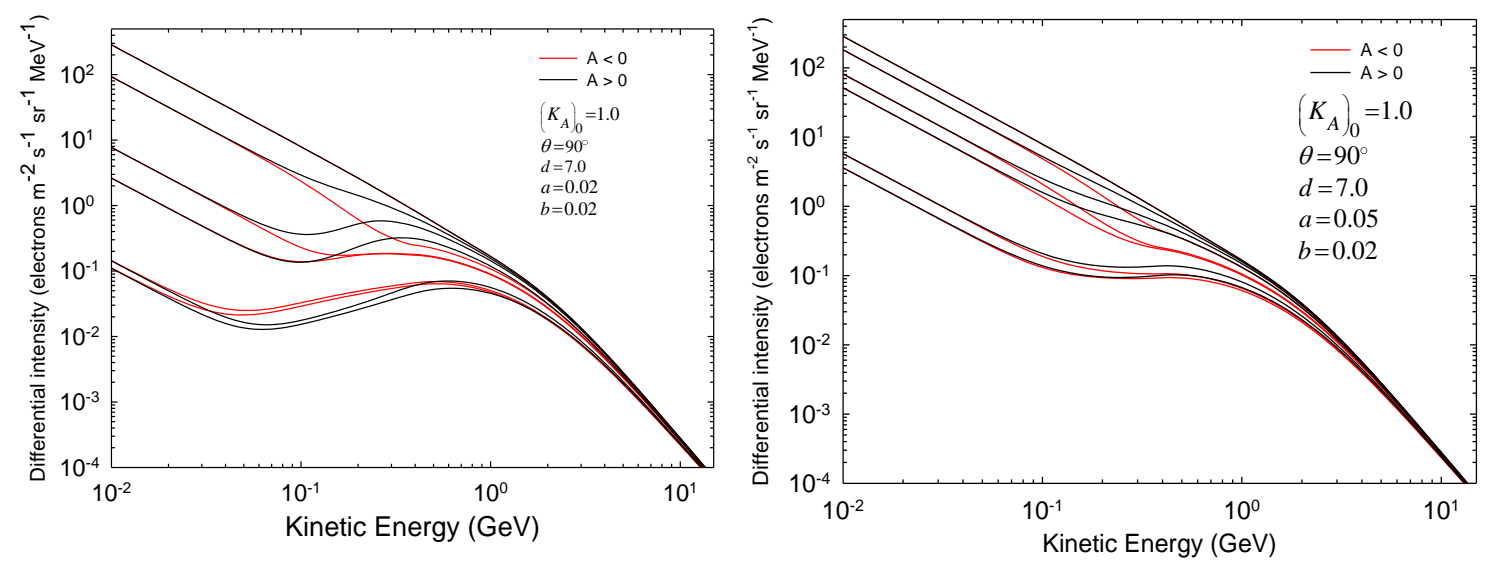

Figure 1: Computed electron spectra as a function of kinetic energy with $\theta=90^{\circ}$ at $1 \mathrm{AU}, 5$ AU, $60 \mathrm{AU} 90 \mathrm{AU}$ and $110 \mathrm{AU}$ with respect to the HPS at $122 \mathrm{AU}$, for both magnetic polarity epochs; the left panel for $K_{\perp r} / K_{\|}=0.02=a$ and the right panel for $K_{\perp r} / K_{\|}=0.05=a$; where $K_{\perp \theta} / K_{\|}=0.02=b$. The enhancement factor is $d=7.0$ and $\left(K_{D}\right)_{0}=1.0$. Note the reduction in the total modulation; at $10 \mathrm{MeV}$ the total reduction in intensity in the left panel is a factor of $\sim 3000$ and in the right panel only $\sim 75$.

\section{Results and discussion}

Using the model discussed in the previous section, the specific aim of this work is to investigate the role of the perpendicular diffusion coefficients in drift reduction for computed electron spectra throughout the heliosphere. This is achieved by first changing the ratio of $K_{\perp r} / K_{\|}$independently of $K_{\perp \theta} / K_{\|}$and secondly by enhancing $K_{\perp \theta}$ through an enhancement factor $d$. [See also 4]. A straight forward rigidity dependence is used, which means that all three DCs have the same rigidity dependence which is constant below $0.4 \mathrm{GV}$ as in Eq. (2). This aspect is important for electron modulation because it causes the total modulation at a given position in the heliosphere to become constant at $E<\sim 50 \mathrm{MeV}$, which is considered as an important feature of electron modulation; see also [15]. 
In Figure 1 the computed electron spectra are showed as a function of kinetic energy, computed with respect to the HPS. The spectra are shown at $1 \mathrm{AU}, 5 \mathrm{AU}, 60 \mathrm{AU}, 90 \mathrm{AU}$ and $110 \mathrm{AU}$ in the equatorial plane $\left(\theta=90^{\circ}\right)$ for both $A>0$ and $A<0$ magnetic polarity epochs, with a tilt angle $\alpha=5^{\circ}$ and with $\left(K_{D}\right)_{0}=1.0$. in Eq. (7). The left panel shows the spectra computed with $K_{\perp r} / K_{\|}=0.02=a$ and the right panel for $K_{\perp r} / K_{\|}=0.05=a$, with the ratio $K_{\perp \theta} / K_{\|}=0.02=b$ unchanged in the equatorial plane. Comparing the left to the right panel, a clear difference between the drift solutions is evident. Drift effect are defined as the difference between $A>0$ and $A<0$ cycles. Note how the drift effects reduce if the total modulation between the HPS (upper curve) and the spectrum at Earth (lowest curve) becomes less, that is when the intensity gradients are correspondingly reduced. For the $K_{\perp r} / K_{\|}=0.05$ case, the drift effects become almost negligible at the Earth, whereas in the outer heliosphere the effects are still there but much smaller. This emphasizes the point that as $K_{\perp r} / K_{\|}$increases, drift effects diminish relatively quickly, making this ratio a most effective way of reducing drift effects indirectly (implicitly) without changing the drift coefficient at all. The question arises to what extend can $K_{\perp r} / K_{\|}$be changed because too little total modulation can easily be caused, as is already apparent in right panel of Figure 1. Unfortunately, diffusion theory cannot yet give a definitive answer. Modeling work found values between $0.005[13,14]$ and 0.05 , but values approaching 0.10 are avoided. To complicate the issue even further, updated diffusion theories indicate that this ratio may not be a constant throughout the heliosphere and also not a constant with rigidity as is assumed here. See earlier work by [16] and more recent work by $[8,17,18]$.

The top panel in Figure 2 shows the function $F(\theta)$ in Eq. (4) in terms of polar angle, with $d=1.0,7.0$ and 13.0, respectively. It enhances $K_{\perp \theta}$ up to a maximum value at the poles as determined by the value of $d$, with no enhancement in the equatorial plane. The region over which the function increases can also be changed to be less steep and to cover a broader range of polar angles. An additional justification for such an increase could be to compensate for a HMF with a meridional component. As long as a Parkerian HMF is used, this enhancement is required [19, 20].

The bottom panel of Figure 2 shows the computed ratios of the $A>0$ and $A<0$ spectra at five different radial distances as described in the figure caption corresponding to three $d$ scenarios shown in the top panel. The ratios are computed for $d=1.0,7.0$ and 13.0 respectively. This summarises the impact that the enhancement of $K_{\perp \theta}$ has on drift effects as a function of energy and radial distances in the equatorial plane; it clearly suppresses drift effects in the heliosphere. Comparing the three solid lines, all for spectra at the Earth, it becomes clear that the maximum drift effects occur for $d=1.0$ and gets reduced with increasing $d$. The same applies for $5 \mathrm{AU}$, with the ratios less than unit for all energies between $\sim 10 \mathrm{MeV}$ and $\sim 10 \mathrm{GeV}$. This also implies that the $A<0$ spectra for electrons are higher than the $A>0$ spectra. The energy at which the maximum drift effects occur moves to somewhat higher values with increasing $d$, indicating that drift effects are first reduced at lower energies. At larger distances from the Sun, the ratios are larger than unity, indicating that the $A<0$ spectra are lower than the $A>0$ spectra. Here, the difference between the blue $(d=7.0)$ and green lines $(d=13.0)$ are indistinguishable, indicating that $d=7.0$ is already causing an optimal reduction in this region of the heliosphere. 
It follows from these computational results that enhancing $K_{\perp \theta}$ is quite effective in reducing drift effects in the inner heliosphere but not in the middle to the outer heliosphere.
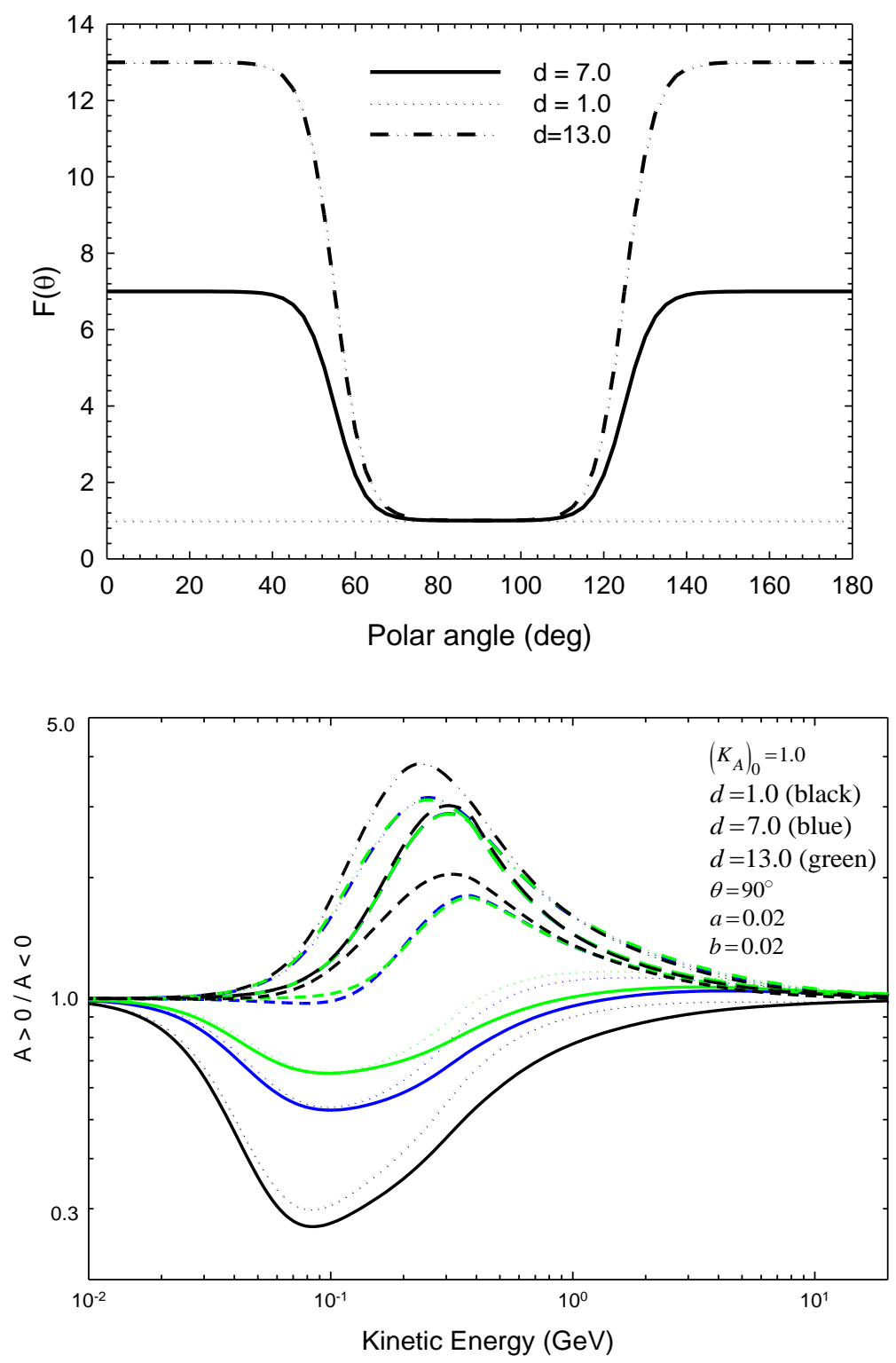

Figure 2: Top panel illustrates how increasing the parameter $d$ from 1.0 to 7.0 to 13.0 enhances the perpendicular diffusin coefficient in the polar direction through $F(\theta)$ in Eq. (4) as function of polar angle $\theta$. The bottom panel shows the computed ratios of $A>0$ and $A<0$ spectra, at $1 \mathrm{AU}$ (solid line), $5 \mathrm{AU}$ (dotted line), $60 \mathrm{AU}$ (shot-dashed line), $90 \mathrm{AU}$ (long-dashed line) and $110 \mathrm{AU}$ (dashed double dotted line) in the equatorial plane with full drifts. Ratios are computed with $d=1.0$ (black lines), $d=7.0$ (blue lines), and for $d=13.0$ (green lines). Deviation from unity is indicative of how large drift effects are. Note how increasing $d$ (black to blue to green) effectively reduces drift effects in the inner heliosphere. 


\section{Discussion and Conclusions}

Drift effects were neglected until [21] and others pointed out that charged particle drift is an important mechanism in CR modulation. The fundamental process of global curvature, gradient and current sheet drifts in the heliosphere is however still not fully understood, especially how HMF turbulence could affect the magnitude of drift on a global scale, and over a full solar activity cycle. General consensus is that implementing weak scattering drift in modulation models is giving too large modulation effects. This follows from the application of these numerical drift models to solar modulation and comparison with observations from the Earth to the outer heliosphere. Particle drift had been recognized as a major modulation process and overshadowed development of advanced diffusion theories until in the early 2000s when major efforts were made to understand diffusion theory better, together with the underlying heliospheric turbulence theory $[22,23]$. It was also then realized that particle drift do not dominate solar modulation over a complete solar cycle but that it is a part of a compelling interplay among the basically four mechanisms, and that this play-off can change over a solar cycle and from one cycle to another [24].

The effects of drift on the global modulation of electrons, and how its reduction influences these effects, were revisited by studying different modulation scenarios using the numerical modulation model. Reducing drift implicitly is a far more subtle process than the strong handed approach of decreasing the drift coefficient directly. This was shown firstly by varying the ratio of $K_{\perp r} / K_{\|}$which is an effective way of reducing drift effects, through changing diffusion.

This ratio should be between 0.005 and 0.05 since making it too large can easily produce too little modulation. To complicate the issue, some updated theories indicate that this ratio may not be constant throughout the heliosphere and also not constant with rigidities as was assumed here. Secondly, the effects of enhancing the diffusion coefficient perpendicular to the HMF in the polar direction were also investigated by studying different enhancement scenarios.

It is found that enhancing $K_{\perp \theta}$ is quite effective in reducing drift effects in the inner heliosphere but not in the middle to the outer heliosphere. The conclusion is made that increasing the ratio of $K_{\perp r} / K_{\|}$and $K_{\perp \theta} / K_{\|}$is most effective in reducing drift effects without changing the drift coefficient at all. 


\section{Acknowledgements}

MSP expresses his gratitude for the partial funding by the South African National Research Foundation (NRF). RRN thanks the NRF and the South African Space Agency (SANSA) for partial financial support during her PhD-study.

\section{References}

[1] Potgieter, M. S. J. Geophys. Res. 101, 24 411, 1996.

[2] Engelbrecht, N. E. and Burger, R. A., Astrophys. J, 772, 46, 2013.

[3] Ngobeni, M. D., PhD, North-West University, South Africa, 2015.

[4] Ngobeni, M. D., and Potgieter, M. S., Adv. Space Res, 53, 11, 1634, 2014.

[5] Ferreira, S. E. S., and Potgieter, M. S., J. Geophys. Res., 107, SSH 12:1-10, 2002.

[6] Potgieter, M. S., J. Geophys. Res., 105 (A8), 18295, 2000.

[7] Raath, J. L. et al. 2015, Solar Phys, submitted .

[8] Ngobeni, M. D., and Potgieter, M. S., Adv. space Res., 48, 300, 2011.

[9] Potgieter, M. S., LRSP, 10, 3, 2013.

[10] Jokipii, J. R., and Kopriva, D. A., Astrophys. J., 234, 384, 1979.

[11] Potgieter, M. S., and Moraal, H. , Astrophys. J., 294, 425, 1985.

[12] Langner, U. W., and Potgieter, M. S., Adv. Space Res., 34,144, 2004.

[13] Ferreira, S. E. S., Potgieter, M. S., Burger, R. A., J. Geophys. Res., 106, 979, 2001.

[14] Ferreira, S. E. S., Potgieter, M. S., Burger, R. A., J. Geophys. Res., 106, 29, 313, 2001.

[15] Potgieter, M. S., and Nndanganeni, R. R., Astrophys Space Sci, 345:33, $2013 \mathrm{a}$.

[16] Burger, R. A., Potgieter, M. S., and Heber, B., J. Geophys. Res., 105, 27447, 2000.

[17] Strauss, R. D., and Potgieter, M. S., J. Geophys. Res., 115, A12111, 2010.

[18] Manuel, R., Ferreira, S. E. S., and Potgieter, M. S., Solar Phys., 289, 2207, 2014.

[19] Ferreira, S. E. S., and Potgieter, M. S., Astrophys, J., 603, 744, 2004a.

[20] Ferreira, S. E. S., and Potgieter, M. S., Adv. Space Res., 34, 115, $2004 \mathrm{~b}$.

[21] Jokipii, J. R., Levy, E. H. and Hubbard, W. B., Astrophys. J., 213(3), 861, 1977.

[22] Bieber, J. W., Adv. Space, Res., 32, 549, 2003.

[23] McKibben, R. B., Adv. Space Res., 35, 518, 2005

[24] Potgieter, M. S., Mwiinga, N., Ferreira, S. E. S., et al., J. Adv. Res., 259, 2013. 